\title{
Agile Erzählungen
}

\section{Rainer Bäcker · Heidi Möller}

Online publiziert: 5. Januar 2022

(C) Der/die Autor(en) 2021

Wenn man sich mit dem Thema „Agilität“ inhaltlich befasst, erscheint es nicht angeraten, es in erster Linie als ein einheitliches theoretisches Konstrukt oder Modell zu betrachten, das man auf seine interne logische Stimmigkeit seiner einzelnen Bestandteile und auf deren empirische Verankerung hin überprüft. Damit würde man Kriterien anlegen, bei denen von vorneherein offensichtlich ist, dass der agile Ansatz dem weder gerecht werden kann noch will. Die eigentliche Bedeutung des agilen Konzepts liegt nicht in seiner theoretischen Stimmigkeit, sondern in seiner praktischen Wirksamkeit. Insofern erscheint es sinnvoll, das uns interessierende Thema Agilität als eine relevante und interessante ,Wirkungseinheit“" (Salber 1969), oder moderner ausgedrückt, als „Erzählung“ in den Blick zu nehmen und zu versuchen, es in seiner inneren Dynamik, seinem Funktionieren und seiner Entwicklung zu verstehen.

Um die unbestreitbare Erfolgsgeschichte und anhaltende Attraktivität der agilen Erzählung nachzuvollziehen, ist es wichtig, sie nicht als ein allgemeingültiges Phänomen in der modernen Arbeitsorganisation zu sehen, sondern sie als eine unter verschiedenen Ausdrucksformen der „Singularisierung“ (Reckwitz 2019) der postindustriellen Ökonomie und Arbeitswelt zu verstehen. Damit wird auch deutlich, dass „New Work“ und der agile Diskurs vor allem die Arbeitsweisen der kulturbestimmenden neuen Akademiker- und Mittelklasse betreffen, während die Arbeitsweisen und -bedingungen der ,neuen Unterklasse“ (Reckwitz 2019), obwohl diese

Dipl.-Psych. Rainer Bäcker

An der Jüch 15, 51465 Bergisch Gladbach, Deutschland

E-Mail: rb@executive-consulting.online

rue du Four, 30190 Sainte-Anastasie, Frankreich

Prof. Dr. Heidi Möller $(\bowtie)$

Institut für Psychologie, Universität Kassel, Holländische Straße 36-38, 34127 Kassel, Deutschland

E-Mail: heidi.moeller@uni-kassel.de 
ungefähr ein Drittel der Gesamtgesellschaft ausmacht, in dieser Erzählung kaum vorkommen und darin bestenfalls ein ebenso randständiges Dasein fristen wie in der gesellschaftlichen Realität.

Unter dieser Perspektive erscheint Agilität eher als eine - neben anderen - Ausdrucksform von grundlegenden Veränderungen und Umbrüchen, die unsere aktuelle Gesellschaft und die damit verbundene Arbeitsorganisation prägen, und weniger als ein einfaches Modell einer neuen Arbeitsweise. Die übergreifenden Themen der neuen Mittelklasse in den postindustriellen Gesellschaften entsprechen von daher auch zentralen Motiven des agilen Mindset, wie z.B.:

- dem Streben nach Selbstverwirklichung und Freiheit einerseits und der Suche nach Sicherheit in der Gemeinschaft/im Schwarm andererseits,

- der Infragestellung von Autoritäten und gleichzeitig dem Streben nach Orientierung gebenden neuen Idolen und Vorbildern,

- dem Bedürfnis nach ideellem ,Sinn“ in der Arbeit versus die permanente Steigerung von Effizienz und Tempo in der Arbeitsgestaltung,

- der Gestaltung einer stabilen, ,einzigartigen“ Identität einerseits und der Entwicklung von allumfassender Anpassungsfähigkeit und Veränderungsbereitschaft andererseits.

Die besondere Wirkkraft der agilen Erzählung scheint zumindest in Teilen darin zu liegen, dass sie das Versprechen beinhaltet, diese, zumindest scheinbaren, Widersprüche zusammenzubringen und miteinander zu versöhnen. Geht es im Kern der agilen Erzählung nicht darum, selbstbestimmtes und „freies“ Arbeiten für alle mit einer neuen Effizienzdynamik der postindustriellen, kapitalistisch organisierten Ökonomie zu verbinden? Von daher ist und bleibt es interessant, zu verfolgen, wie sich die zentralen Motive der agilen Erzählung bei ihrem „Realitäts-Check“ in der täglichen praktischen Arbeitsgestaltung weiter ausgestalten und wie sich dabei neue (Erzähl-) Versionen entwickeln und Lösungen für die immanenten Spannungsfelder ausbilden.

Mit dieser Ausgabe der OSC wollen wir einen Eindruck von der Vielgestaltigkeit dieser agilen Erzählung vermitteln und einen Beitrag zu deren Weiterentwicklung leisten.

Den Auftakt macht dabei Robert Erlinghagen, der uns einen Überblick zu der Welt des Agilen gibt und dabei insbesondere auf deren inneren Spannungsverhältnisse eingeht. Für ihn bedeutet Agilität in Anlehnung an Stefan Fischer vor allem Anpassungsfähigkeit (Fischer 2016). Unter dieser Perspektive stellt er die Entwicklung des agilen Ansatzes dar und lotet aus, wie verantwortungsvolle Organisationsberater/innen, Supervisor/innen und Coaches in diesem Kontext sinnvoll tätig werden können.

Die Themen von Erlinghagen finden sich zum Teil in den Ergebnissen einer psychologischen Studie wieder, die Rainer Bäcker im nächsten Beitrag vorstellt. Mithilfe von psychologischen Tiefeninterviews wurde dabei das agile Mindset befragt. In den Interviews wurden einige paradoxe Zusammenhänge deutlich, mit der sich die psycho-logischen Zusammenhänge des Agilen beschreiben lassen. Von diesen Erkenntnissen ausgehend, werden erste Ableitungen für eine „,dynamische Führung“ in einem agilen Kontext formuliert. 
In der Ausarbeitung von Martina Musati wird der Einsatz des agilen Konzepts im Rahmen der Reformkonzepte öffentlicher Verwaltungen diskutiert. Es wird aufgezeigt, dass durch diesen Ansatz auch in einem Kontext, den man nicht zwangsläufig mit einem agilen Mindset verbindet, neue Möglichkeiten eröffnet werden können. Dabei wird den Führungskräften und dem Modell „Agile Five“ eine zentrale Rolle zugesprochen.

Die Thematik von Agilität und Führung steht auch im Zentrum der Überlegungen, die Heidi Möller und Thomas Giernalczyk anstellen. Sie machen deutlich, vor welchen Herausforderungen Führungskräfte, die an Macht und Einfluss verlieren, in agilen Organisationen stehen. Unter dem Stichwort „New Leadership“ zeigen sie auf, wie mithilfe des Konzepts der psychologischen Sicherheit Führungskräfte in diesem Umbruch Handlungsspielräume gewinnen und ihr Selbstmanagement ausbauen können.

Die Möglichkeiten, aber auch die Schwierigkeiten und Grenzen, die sich bei der Umsetzung des agilen Konzepts in der Praxis zeigen, werden in den Schilderungen von Pierre-Pascal Urbon und Jörg Staff anschaulich vermittelt.

Pierre-Pascal Urbon beschreibt, wie er als Vorstandsvorsitzender des ITK-Distributors und Dienstleisters KOMSA mithilfe agiler Methoden das größte ChangeProjekt seit Unternehmensgründung durchführte. Dabei wurden die zentralen Unternehmensprozesse verschlankt und eine neue Führungs- und Steuerungsstruktur aufgebaut.

Im Beitrag von Jörg Staff, Vorstand und Chief People Officer der Atruvia AG, erfahren wir, wie bei der Atruvia ein unternehmensweiter agiler Transformationsprozess umgesetzt wurde. Der Autor nimmt uns dabei mit auf eine von ihm bezeichnete „disruptive Transformationsreise“. Dabei wird deutlich, wie wichtig es ist, nicht nur die Arbeitsweisen in den Mitarbeiterteams zu verändern, sondern auch ein neues Zusammenarbeits- und Führungsverständnis zu etablieren, das auch das Top-Management und die Vorstände mit einbezieht.

Unsere agile Erzählung findet ihren Abschluss mit einem neuen Format, das wir für die OSC kreiert haben. Unter der Moderation von Thomas Bachmann haben sich im November einige Herausgeber/innen und wissenschaftliche Beiräte zu einem Online-Gespräch über New Work und Agilität getroffen. Das Transkript der Diskussion macht anschaulich deutlich, unter welch unterschiedlichen Perspektiven Agilität betrachtet und bewertet werden kann.

Der freie Beitrag der Zeitschrift fokussiert das Potenzial positiver Psychologie in der Arbeitswelt. Carmen Elisabeth Lobbe und Kathrin Reinke stellen differenziert den Wissensstand zum Nutzen der positiv psychologischen Interventionen in der Arbeitswelt dar und geben Einblick in eine eigene Untersuchung. Diese zeigt, dass Wohlbefinden von Arbeitsnehmer/innen und Ressourcenaktivierung mehr braucht als Kurzinterventionen.

In der Rubrik Kulturanalysen wird der Film „Skin“ von Regisseur Guy Nuttiv (2018) durch Alexander Hagemann, Nathalie Lehmann und Kim Marsh besprochen. Im Film werden Radikalisierungs- und Deradikalisierungsprozesse der rechtsextremistischen Szene um die authentische Lebensgeschichte des Bryon in den USA erzählt. Die Autor/innen stellen empirische Ergebnisse vor, die sie als im Film gut in Bilder gesetzt beschreiben. Sie skizzieren zudem Wege heraus aus der rechten 
Szene und beschreiben die Rolle, die Beratung dabei spielen kann. Der Beitrag mag exemplarisch dafür stehen, wie Radikalisierung auch in anderen Kontexten erklärbar ist und welche Gelingensbedingungen es für den Ausstieg braucht. Unschwer ist zu erkennen, dass die theoretischen Zugänge nutzbar sind, um auch die sich zuspitzende Dynamik von Verschwörungstheoretiker/innen und Querdenker/innen psychologisch nachzuvollziehen.

Funding Open Access funding enabled and organized by Projekt DEAL.

Open Access Dieser Artikel wird unter der Creative Commons Namensnennung 4.0 International Lizenz veröffentlicht, welche die Nutzung, Vervielfältigung, Bearbeitung, Verbreitung und Wiedergabe in jeglichem Medium und Format erlaubt, sofern Sie den/die ursprünglichen Autor(en) und die Quelle ordnungsgemäß nennen, einen Link zur Creative Commons Lizenz beifügen und angeben, ob Änderungen vorgenommen wurden.

Die in diesem Artikel enthaltenen Bilder und sonstiges Drittmaterial unterliegen ebenfalls der genannten Creative Commons Lizenz, sofern sich aus der Abbildungslegende nichts anderes ergibt. Sofern das betreffende Material nicht unter der genannten Creative Commons Lizenz steht und die betreffende Handlung nicht nach gesetzlichen Vorschriften erlaubt ist, ist für die oben aufgeführten Weiterverwendungen des Materials die Einwilligung des jeweiligen Rechteinhabers einzuholen.

Weitere Details zur Lizenz entnehmen Sie bitte der Lizenzinformation auf http://creativecommons.org/ licenses/by/4.0/deed.de.

\section{Literatur}

Fischer, S. (2016). Definition: Agilität als höchste Form der Anpassungsfähigkeit. https://www.haufe.de/ personal/hr-management/agilitaet/definition-agilitaet-als-hoechste-form-der-anpassungsfaehigkeit_ 80_378520.html. Zugegriffen: 16.11.2021.

Reckwitz, A. (2019). Die Gesellschaft der Singularitäten. Berlin: Suhrkamp.

Salber, W. (1969). Wirkungseinheiten. Kastellaun, Wuppertal: A. Henn. 